\title{
FATIGUE BEHAVIOR OF LIGHTLY REINFORCED CONCRETE BEAMS IN FLEXURE DUE TO OVERLOAD
}

\author{
NAGESH H.E ${ }^{*}$, G. APPA RAO ${ }^{\dagger}$ \\ *Indian Institute of Technology Madras, Chennai-600036, India \\ e-mail: nageshhe@gmail.com \\ $\dagger$ Indian Institute of Technology Madras, Chennai-600036, India \\ e-mail: garao@iitm.ac.in
}

Key words: Overload, Fatigue, Size effect, RC beams, Lightly reinforced

\begin{abstract}
This paper reports experimental investigations on the fatigue behavior of lightly reinforced concrete (RC) beams subjected to an overload at different instants of loading. Effect of size on fatigue response of $\mathrm{RC}$ beams has also been studied. A review of existing code provisions and equations proposed by various researchers to predict fatigue life has been reported. Crack growth mechanism in steel reinforcement and concrete, mode of failure and mid-span deflection of beams have been discussed. It has been observed that overload at any instant decreases the fatigue life of RC beams by accelerating crack initiation process. The fatigue life of lightly RC beams has been observed to be influenced by the size.
\end{abstract}

\section{INTRODUCTION}

Concrete pavements and upper deck slab of box-girder bridges are few examples of lightly $\mathrm{RC}$ structures. These members are subjected to routine, repetitive loading due to movement of vehicular traffic. This loading causes progressive, permanent internal damage in its constituent materials. Under service loads, failure process is mainly initiated by microcracking in concrete. Microcracks coalesce to form macrocracks at higher levels of traffic or impact or accidental overloads. An overload widens the crack and intensifies the level of damage. Hence, it is important to understand the behavior of overstressed members and its effect on fatigue life.

Generally, fatigue failure in RC members is caused by fatigue fracture of steel reinforcing bars. Moreover, applied stress range has a significant influence on the fatigue life of reinforcing bar.
Most of the current models depend on empirical S-N (Stress - Number of cycles) approach to predict the fatigue life [1-5]. The conventional S-N approach requires extensive experimental data, which is based on the stress parameter only. However, application of fracture mechanics principles to study the fatigue fracture parameters in $\mathrm{RC}$ beams has gained importance in recent years.

Chen and Cheng [6] defined fatigue failure in $\mathrm{RC}$ beams based on unstable propagation of an effective crack in concrete. Cohesive model was used in their study, for providing a rational approach for predicting fatigue life. Sain and Kishen [7] evaluated the residual strength of $\mathrm{RC}$ beam by combining Paris equation and Rcurve approach. Peerapong and Matsumoto [8] performed fatigue failure analysis in RC slabs based on bridging stress degradation relationship for cementitious materials. Stress degradation was considered as a principal cause for crack propagation in their study [8]. 


\section{MATERIAL BEHAVIOR UNDER FATIGUE LOADING}

\subsection{Fatigue of concrete}

The effect of repeated loading develops a continuous microcracking process between hydrated cement paste and aggregate interfaces in concrete. Further, it causes macroscopic detrimental and permanent changes in its mechanical properties. Most of the nonlinear material models assume fracture process zone (FPZ) is responsible for this variation in material properties [9]. The degradation of this aggregate bridging process results in crack growth in concrete.

Flexural fatigue strength of concrete has been found to be size dependent [10-11]. Fatigue testing on concrete beams under same flexural stress found that smaller beam has longer fatigue life than larger beam [12].

\subsection{Fatigue of reinforcing bars}

The fatigue strength of steel reinforcing bar is relatively lower compared to its static strength. Fatigue life of reinforcing bar is mainly influenced by bar geometry, deformations on bars, lugs, ribs and defects. Fatigue life is also affected by the type of steel, size of the bars, chemical composition, microstructure and nature of loading cycles [2, 13]. Moreover, the fatigue strength of reinforcing steel bar is not proportional to its static yield capacity [14]. Cyclic loading causes high local stress concentrations near ribs or defects which are sufficient to initiate micro cracking. These microcracks grow to a critical length under repetitive loading leading to fatigue fracture.

\subsection{Fatigue of reinforced concrete beams}

Fatigue behavior of axially loaded reinforcing bar in the air and plain concrete differs significantly. Moreover, fatigue performance of RC beams involves composite action between steel and concrete. The interaction between steel and concrete under cyclic loading is a complex process and difficult to model [15]. Flexural cracks in RC beam produce local stress concentrations in bars in its plane. The defect in reinforcing steel bar is unlikely to coincide with the crack plane in concrete. This causes the rebar to initiate its failure process in nearby cracked concrete location. Hence, in RC beams crack in concrete decides the fatigue failure in bar resulting longer fatigue life.

Generally in RC beam steel governs the fatigue life. Fatigue failure of concrete is delayed in RC beams because of its ability to redistribute compressive stresses. Under high compressive stress, only fatigue failure of concrete may be expected in RC beams $[1,15]$.

In recent years, studies based on structural size effect have gained importance. It is well known that the fatigue of plain concrete in bending exhibits size effect [10,12]. This clearly shows that there is a need to study the fatigue behavior of RC beams with different sizes. Hence, the present study was undertaken.

\section{EFFECT OF OVERLOAD}

In metals overload causes compressive residual stress in the crack tip plastic zone and crack closure in the plastic zone leads to smaller effective stress ranges. This increase in the plastic zone size ahead of the crack tip retards crack growth and increases the fatigue life [1617].

In concrete, an overload causes an increase in the size of fracture process zone. Unlike in metals, FPZ in concrete accelerates the rate of crack propagation and decreases fatigue life [18]. FPZ in concrete exhibits more sensitivity to fatigue loading of concrete than uncracked ligament [9]. The studies on effect of overload in fatigue life of $\mathrm{RC}$ beams is limited in the literature, which motivates the present study.

\section{REVIEW OF CODE PROVISIONS AND MODELS}

ACI 215 [13] limits maximum service level stress range in equation (1) than estimating fatigue life. It also recommends the permissible 
stress range of $138 \mathrm{MPa}$ in reinforcing bar, whereas, AASHTO [19] recommends the permissible stress range limit in bars as 162 $\mathrm{MPa}$. It also limits the stress range in steel using equation (2) where, $r / h$ is the ratio of the steel base radius to the height of the rolled-on transverse deformations. The Canadian highway bridge design code [20] restricts the maximum stress range in straight bars to 125 MPa. CEB-FIP Model Code [21] predicts the fatigue life using equation (3) using S-N relationship in for bar diameter less than 16 $\mathrm{mm}$. In Table 1 the equations for predicting fatigue life are tabulated against corresponding code or researcher.

Table 1: Equations for predicting fatigue life

\begin{tabular}{ll}
$\begin{array}{l}\text { Code/ } \\
\text { Authors }\end{array}$ & \multicolumn{1}{c}{ Equation } \\
\hline ACI 215 [13] & $\sigma_{r}=161-0.33 \sigma_{\min }$
\end{tabular}

Where $\sigma_{r}$ and $S_{r}$ are stress range in tension steel, $\varepsilon_{r}$ is strain range, $\sigma_{\min }$ is minimum stress range in steel bar and $\mathrm{N}$ is the number of cycles.

Along with code provisions, many researchers proposed equations to estimate fatigue life. The equation (4) proposed by Moss [5] estimates fatigue life based on bending tests of reinforcing bars embedded in concrete, where in equation (4), $\mathrm{m}$ is an empirical constant of value 8.7; and $\mathrm{K}$ for mean line of the relationship $=0.11 \times 10^{29}$ and for mean minus two standard deviations $=0.59 \times 10^{27}$. Tilly and Moss [3] modified equation (4) of Moss [5] based on steel rebars tested in the air and proposed equation (5) where $\mathrm{m}=9 ; \mathrm{k}^{*}=0.75 \times$ $10^{27}$ for rebars of diameter less than $16 \mathrm{~mm}$ and $\mathrm{k}^{*}=0.007 \times 10^{27}$ for rebars of diameter greater than $16 \mathrm{~mm}$.

Helgason and Hanson [1] derived equation (6) based on a statistical analysis and also shown that lowest stress range that cause fatigue failure in the reinforcing steel was 165 MPa. Papakonstantinou et al. [22] also given equation (7) to estimate the fatigue life based on regression analysis. The equation (8) proposed by Zhao [23] relates fatigue life with strain in tension steel.

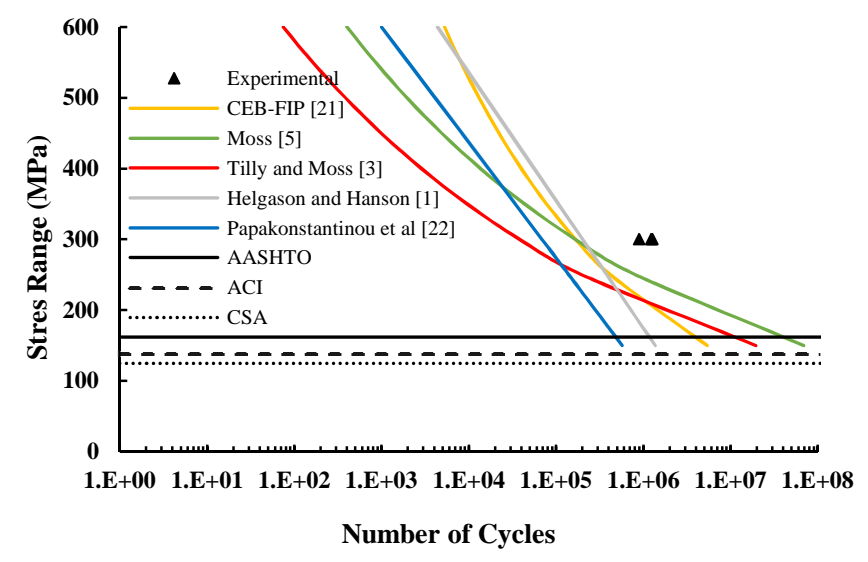

Figure 1: Comparison of fatigue life prediction models

Code provisions and models developed by researchers to predict fatigue life are compared in Figure 1. Experimental results show that the fatigue life has been underestimated by these models. Hence, there is a need to develop a model which can estimate the fatigue life accurately.

\section{EXPERIMENTAL PROGRAM}

The experimental program consists of casting eight RC beams. Concrete materials and mix proportions, dimensions of beams and reinforcement details are discussed below.

\subsection{Materials and mix proportions}

The concrete mix was produced by using 53 grade ordinary Portland cement, $10-\mathrm{mm}$ graded coarse aggregate and river sand as fine aggregate. The specific gravity and fineness 
modulus of fine aggregate were 2.78 and 2.82 respectively. Potable water of $\mathrm{pH} 7.5$ was used for mixing in concrete and curing of specimens. The concrete mix proportion used was 1: 1.84: 2.75 respectively cement: fine aggregate: coarse aggregate. The cement content was 400 $\mathrm{kg} / \mathrm{m}^{3}$ and the water-to-cement ratio was 0.39 . The compressive strength of concrete after 28 days on $150 \mathrm{~mm}$ size standard cubes was 60.7 $\mathrm{MPa}$ with a standard deviation of $4.3 \mathrm{MPa}$. The steel bar of nominal diameter $8 \mathrm{~mm}$ was used in all specimens having a yield strength of 547 $\mathrm{MPa}$ and ultimate strength of $634 \mathrm{MPa}$. The fracture energy test was carried out according to RILEM standard [24]. The fracture energy $\left(\mathrm{G}_{\mathrm{f}}\right)$ was found to be $97.2 \mathrm{~N} / \mathrm{m}$.

\subsection{Specimen description and reinforcement details}

The depth of the beam is varied from 150 $\mathrm{mm}$ to $600 \mathrm{~mm}$ by maintaining width $(150 \mathrm{~mm})$ constant. The span between the supports is six times the depth of beam and the spans are 900 $\mathrm{mm}, 1800 \mathrm{~mm}$ and $3600 \mathrm{~mm}$ for small, medium and large beams respectively.

All beams were designed as singly reinforced with tension reinforcement $(0.25 \%)$ percentage. In order to overcome the influence of size of reinforcing bars on fatigue loading same diameter of reinforcing bars were adopted. In Table 2 beam dimensions and reinforcement details are shown.

Table 2: Beam dimensions and reinforcement details

\begin{tabular}{ccc}
\hline $\begin{array}{c}\text { Beam } \\
\text { designation }\end{array}$ & $\begin{array}{c}\text { Depth } \\
(\mathbf{m m})\end{array}$ & $\begin{array}{c}\text { Number- } \\
\text { diameter of bar }\end{array}$ \\
\hline SB-M & 150 & $\# 1-8 \mathrm{~mm} \varphi$ \\
\hline MB-M & 300 & $\# 2-8 \mathrm{~mm} \varphi$ \\
\hline LB-M & 600 & $\# 4-8 \mathrm{~mm} \varphi$ \\
\hline SB-F & 150 & $\# 1-8 \mathrm{~mm} \varphi$ \\
\hline SB-OF & 150 & $\# 1-8 \mathrm{~mm} \varphi$ \\
\hline SB-FO & 150 & $\# 1-8 \mathrm{~mm} \varphi$ \\
\hline MB-F & 300 & $\# 2-8 \mathrm{~mm} \varphi$ \\
\hline LB-F & 600 & $\# 4-8 \mathrm{~mm} \varphi$ \\
\hline
\end{tabular}

The nomenclature is described below,
$\mathrm{SB}$ - small beam, MB - medium beam, LB - large beam, $\mathrm{M}$ - monotonic loading, $\mathrm{F}$ - fatigue loading, $\mathrm{OF}$ - initial overload FO - an overload at three lakh cycles.

A notch was made in the beam bottom face during casting by placing a steel plate at the midspan. The notch is located at the center of the beam in the bottom cover as shown in Figure 2. The notch was executed to measure crack mouth opening displacement and the strain in tension reinforcing bar at notch plane. The depth of notch is $10 \mathrm{~mm}, 20 \mathrm{~mm}$ and 40 $\mathrm{mm}$ for small, medium and large beam specimens respectively. The distance between the bottom edge of the beam to tension reinforcement is one-tenth of overall depth in all beams.

\section{EXPERIMENTAL SETUP AND INSTRUMENTATION}

Figure 2 shows the three-point bend loading set-up for both monotonic and fatigue tests. The beams were simply supported with one end of the beam pinned and the other end with a roller support. To avoid accidents in case of out-ofplane movement, vertical steel rigs were placed at the supports. This arrangement was made such that rigs were not in contact with the concrete beam surface. The actual test setup for fatigue loading is shown in Figure 3.

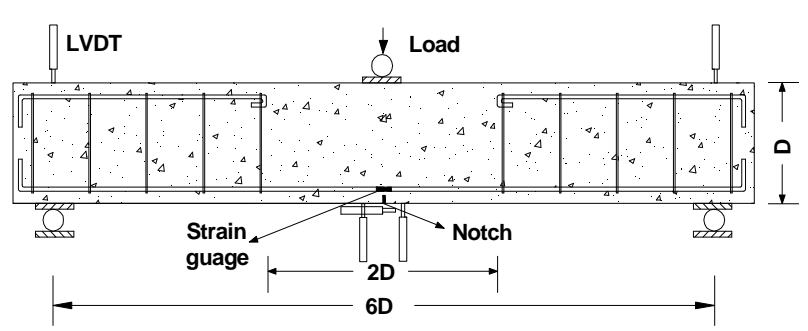

Figure 2: Test setup and specimen details

The beam specimens were instrumented with strain gauges and linear variable displacement transducers (LVDTs). Strain gauges were mounted on the tensile reinforcing bar prior to casting of concrete in the midspan. A load cell was placed between beam and actuator or hydraulic jack to measure the 
applied load.

A pair of LVDTs was placed at the center on either side of the notch to record the mid-span deflection. LVDTs were placed horizontally on top and bottom surface of the beam to measure strain. Crack mouth opening displacement (CMOD) was measured using LVDT, such that a small gauge length was preferred. To record mortar settlements at the supports, vertical LVDTs were mounted on the top face of the beam above the supports. Crack widths were measured using optical microscope having a scale division of $0.02 \mathrm{~mm}$. The crack tip was located carefully using a magnifying lens and crack length was measured using a digital Vernier scale of least count $0.01 \mathrm{~mm}$. The instrumentation arrangements as shown in Figure 2 was used for both monotonic and fatigue tests.

\section{TESTING PROCEDURE}

\subsection{Monotonic testing}

A closed loop servo controlled hydraulic actuator of capacity $500 \mathrm{kN}$ was used for flexural loading. The monotonic load was applied under deflection control at a rate of $0.002 \mathrm{~mm} / \mathrm{sec}$ up to the cracking load, and then at $0.01 \mathrm{~mm} / \mathrm{sec}$ to completion. Monotonic tests were performed to establish the cracking load, yield load capacity of similar RC beams to be tested in fatigue.

\subsection{Fatigue testing}

The load ranges for fatigue tests were arrived based on monotonic flexural tests. The load ranges between $10 \%$ and $65 \%$ of monotonic yield load on corresponding beams. The minimum fatigue load was ensured to avoid impact loading on beam and to ensure the stability of test set-up. Final load ranges were obtained such that minimum and maximum stress in reinforcement bar was same in all the beams. Self-weight of the beam has also been accounted. However, only one stress level (300 $\mathrm{MPa}$ ) was considered to study the fatigue behavior of all specimens.

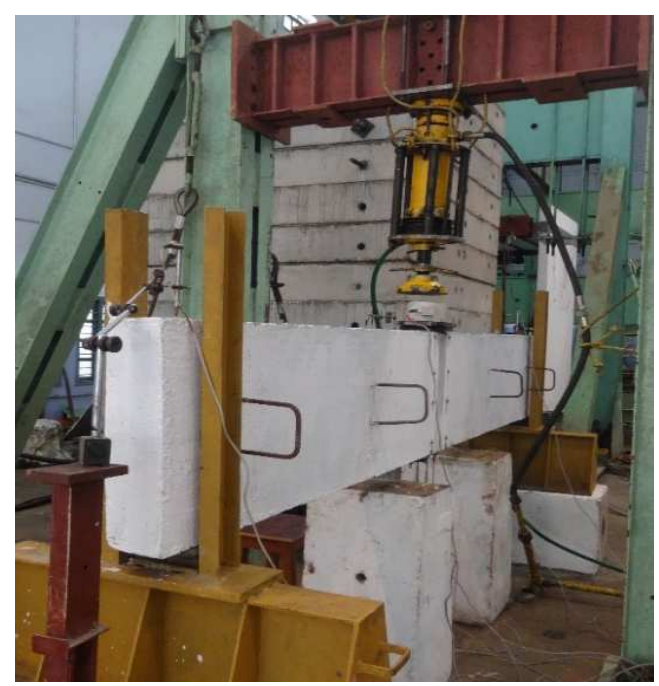

Figure 3: Fatigue test setup

The constant amplitude fatigue load was applied using load controlled hydraulic jack of capacity $200 \mathrm{kN}$. During fatigue testing initially, the load was applied monotonically up to the median load of predetermined load range. The cyclic load was then applied using sinusoidal wave, and corresponding maximum and minimum loads were fixed. The frequency of load cycles was limited to $3 \mathrm{~Hz}$ to avoid any overheating of the reinforcing bar [25] and to avoid hysteresis effects [22]. The tests were continued till the failure of all reinforcing bars. Testing was performed continuously for 4-5 days without interruption up to failure.

Data logging was set to record data continuously throughout the test till failure. While, the data was saved only at a convenient number of intervals for 60 seconds at a rate of $200 \mathrm{~Hz}$.

\subsubsection{Application of overload}

In order to simulate severe overloading condition, a maximum load up to the yielding of steel (yield load) was applied. Before the application of an overload, fatigue loading was terminated. The beam was then loaded monotonically to its corresponding yield load and then unloaded. The cyclic loading was then resumed to its previous constant amplitude load level and maintained the same throughout the test till failure. During overloading, crack 
developments, crack widths and Demec gauge readings were recorded.

\section{MONOTONIC TEST RESULTS AND DISCUSSIONS}

Beams tested under monotonic loading failed by rupture of tension reinforcement after long deformation. Such ductile failure was due to the low percentage of tensile steel reinforcement. Lightly reinforced concrete beam has a characteristic of predominant single crack flexural failure. Cracking load, yield load and ultimate load of all beams were recorded. In case of large beam (LB-M) few cracks were observed before final failure.

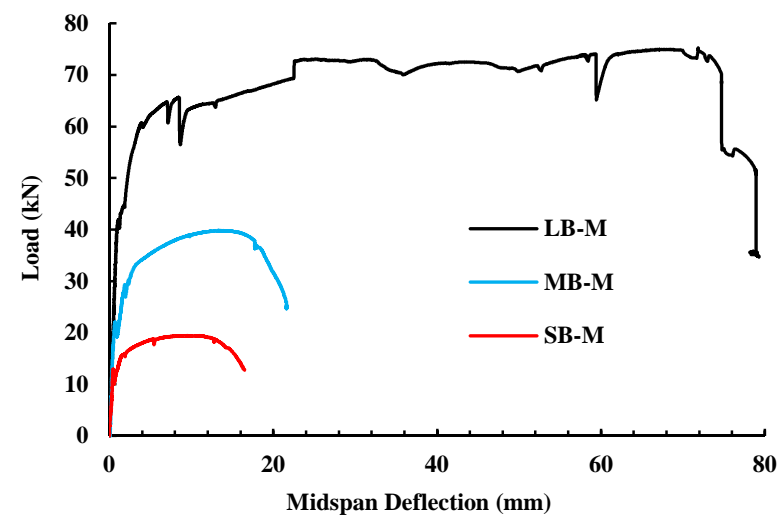

Figure 4: Load versus midspan deflection

Figure 4 show the load versus the midspan deflection response of small, medium and large beams. From Figure 4 it is observed initial linear elastic response up to concrete cracking in all beams. At peak due to concrete cracking a sudden drop in load was noticed, after which gradual increase in further load with displacement was observed. This behavior followed by horizontal plateau, indicates perfectly plastic behavior.

\section{FATIGUE TEST RESULTS AND DISCUSSIONS}

The summary of fatigue test results are shown in Table 3. The number of cycles which caused the crack initiation in concrete and final failure of tensile steel reinforcing bars which corresponds to the ultimate failure of beams are tabulated.

Table 3: Fatigue test summary

\begin{tabular}{ccc}
\hline $\begin{array}{c}\text { Specimen } \\
\text { designation }\end{array}$ & $\begin{array}{c}\text { Number } \\
\text { of cycles } \\
\text { to crack }\end{array}$ & $\begin{array}{c}\text { Number of } \\
\text { cycles to } \\
\text { failure }\end{array}$ \\
\hline SB-F & 6900 & $12.19 \times 10^{5}$ \\
\hline SB-OF & 1 & $3.95 \times 10^{5}$ \\
\hline SB-FO & 5600 & $6.35 \times 10^{5}$ \\
\hline MB-F & 3700 & $12.66 \times 10^{5}$ \\
\hline LB-F & 5300 & $8.89 \times 10^{5}$ \\
\hline
\end{tabular}

\subsection{Mode of failure}

The failure observed in all beams was due to fatigue fracture of longitudinal tensile reinforcing bars. Failure of steel bars was noticed in the pre-notched location of concrete beam. Single flexural crack in concrete was originated from the notch tip after few thousand cycles and propagated gradually till failure. At the onset of failure, unstable crack propagation was observed. All beams failed due to flexural crack opening (Mode I). It was also noticed that once a crack formed, no significant changes in the crack pattern was observed despite with an increased number of cycles.

\subsection{Failure mechanism in reinforcing bar}

Failure mechanism in the bar was characterized by crack initiation (stage 1 ), crack propagation (stage 2) and rupture phase (stage $3)$ as indicated in Figure 5.

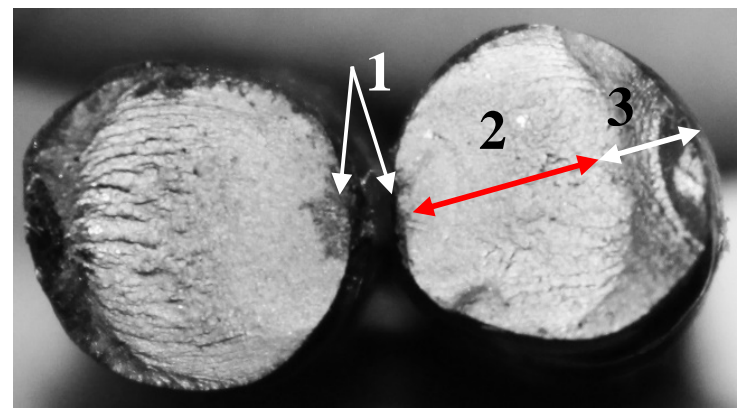

Figure 5: Fatigue fractured surface of reinforcing bar.

Cracks normally originated from high stress concentration locations such as ribs corner, rib intersection, intersection of transverse lugs and 
longitudinal ribs and also from defects on the surface of the bars. This crack initiation was followed by a stable crack growth. After the crack propagation had reached to its critical depth, sudden fracture was observed. The critical crack depth was observed to be $0.55 \mathrm{~d}$ to $0.68 \mathrm{~d}$ where ' $\mathrm{d}$ ' is the diameter of the bar.

\subsection{Microscopic observations}

In the case of LB-F beam fatigue failure of one reinforcing steel bar led to the significant increase in stress levels in the remaining bars. The failure resulted in those bars was of low cycle, high stress fatigue. It was observed that these later fractured surfaces had a clear distinction from the first fractured bar. Hence, the fatigue failure surface was observed using scanning electron microscopy (SEM). SEM micrographs of fracture surfaces of the first fractured bar and the later bar are shown in Figure 6 and Figure 7 respectively.

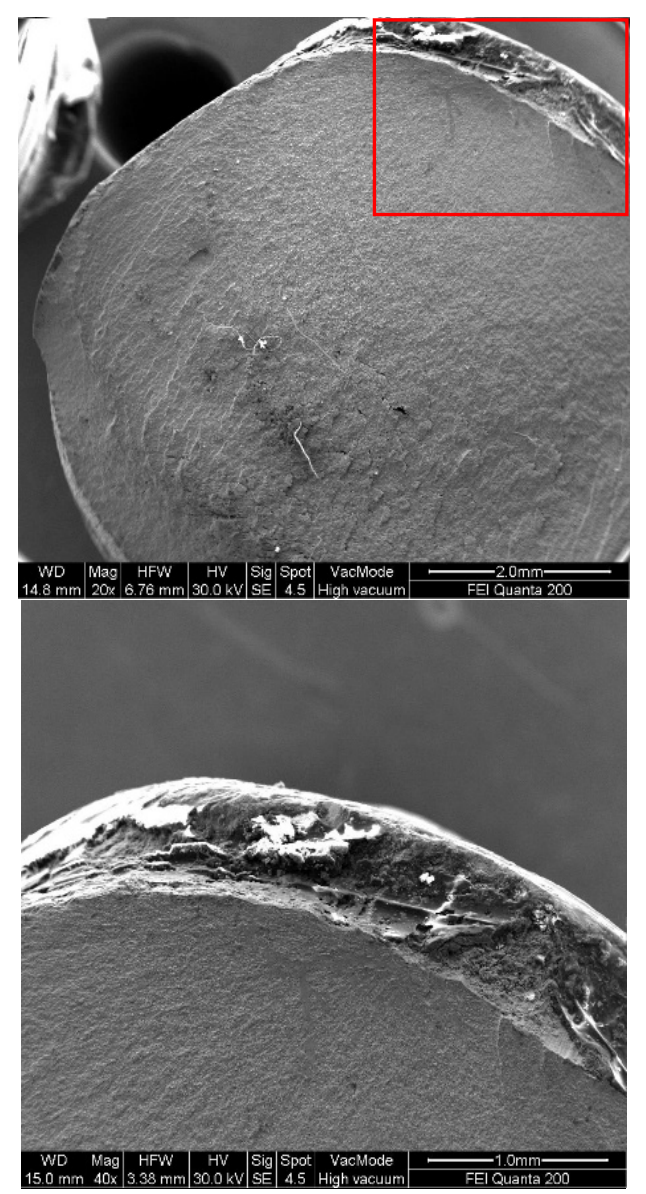

Figure 6: SEM micrographs of failure surface of first fractured bar
In Figure 6 the crack initiation, propagation and fracture can be observed. However, in the case of later fractured bar (Figure 7) the failure was neither cup and cone fracture as in the case of static failure nor typical fatigue failure. Figure 7 also shows the defect which caused the fracture. It can also be observed from Figure 7 the diameter of reinforcing bar reduced to 4.5 $\mathrm{mm}$.

\subsection{Crack growth in concrete}

In order to monitor crack tip during fatigue loading, both sides of the beam were cleaned with acetone and a thin coating of water soluble white paint was applied. The typical crack growth history of the beam LB-F is shown in Figure 8 is discussed here. The crack lengths reported were observed visible crack tip, which does not represent traction free crack length.

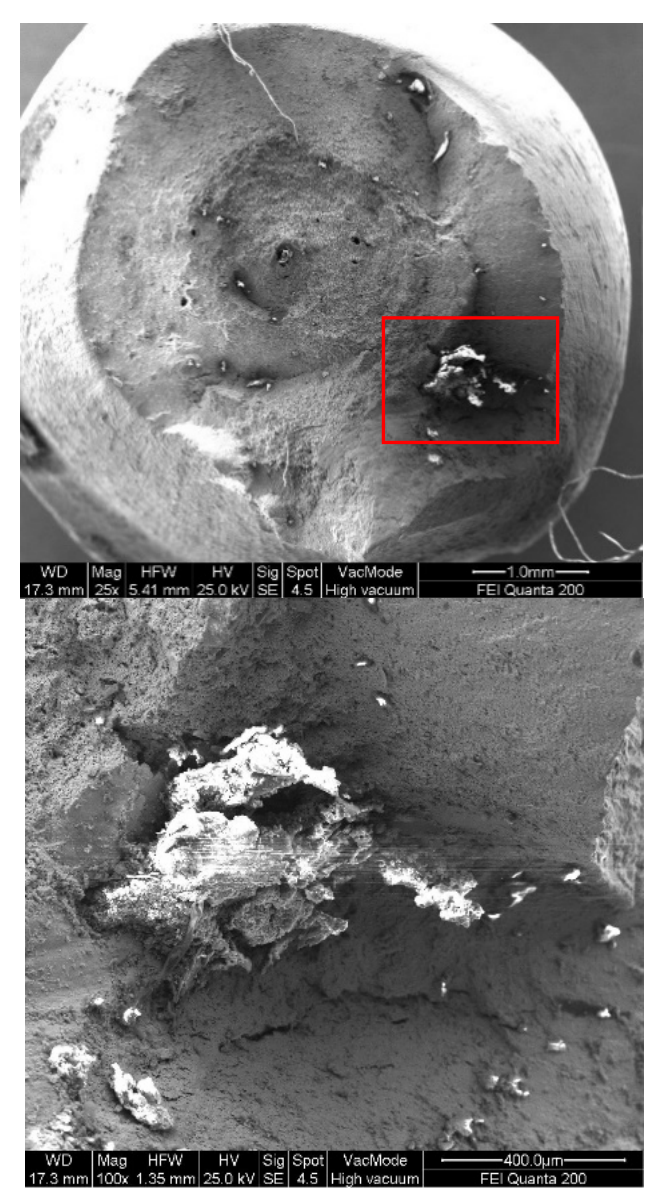

Figure 7: SEM micrographs of failure surface of later fractured bar 
Initially, no crack was observed up to 5300 cycles. Suddenly a crack originated from notch tip which led to an instantaneous increase in crack length as observed in Figure 8. As the fatigue loading was continued, a gradual increase in the crack growth was observed over few hundred thousand cycles. No significant crack growth was noticed once the crack reached neutral axis. Rapid increase in crack growth was observed after fatigue fracture of the first bar as noticed in Figure 8. This behavior followed by unstable crack propagation in concrete causing final failure.

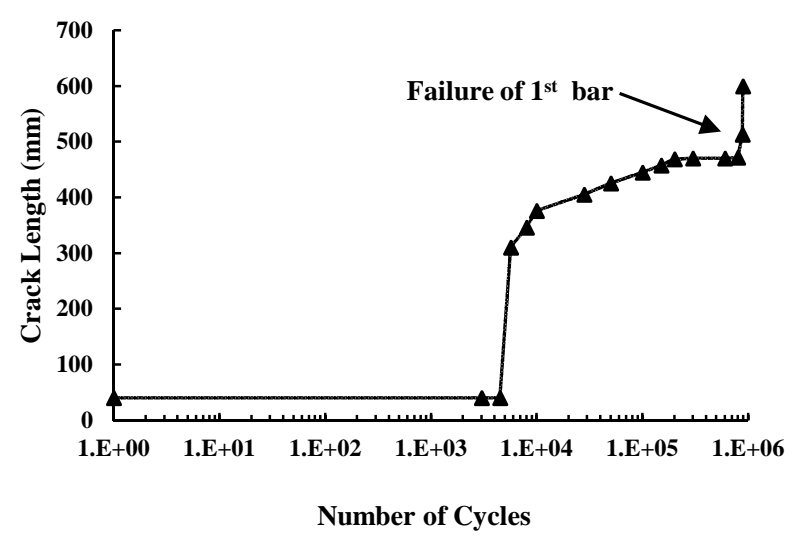

Figure 8: Crack length versus number of cycles of LB-F

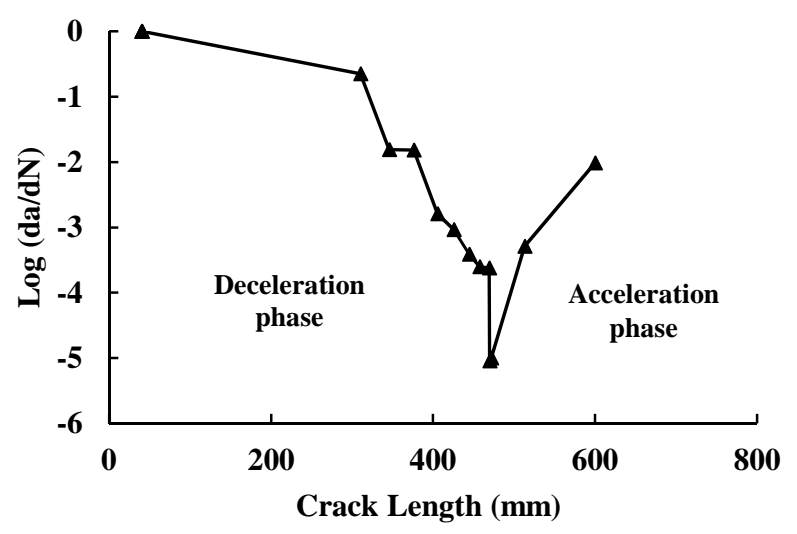

Figure 9: $\log (\mathrm{da} / \mathrm{dN})$ versus crack length of LB-F

The response between $\log (\mathrm{da} / \mathrm{dN})$ versus crack length of LB-F beam (Figure 9) shows the deceleration phase and acceleration phase. In deceleration phase, the rate of crack growth decreases with increase in crack length. This clearly shows that the resistance offered for crack growth increases with increase in crack length. Near the end of deceleration phase, over a period no appreciable increase in crack length was observed with increase in number of cycles. The fatigue fracture of first reinforcing bar resulted in an increase of crack length which led to the acceleration phase. It was also found that an increase in the rate of crack growth caused the final failure.

\subsection{Strain in tensile reinforcement}

At the location of the notch, local strains in the reinforcement bar were measured using electrical resistance strain gauges mounted to it. The variation of strain in steel reinforcement at maximum load versus number of cycles for SB$\mathrm{F}$ beam is plotted in Figure 10. A rapid increase in strain in steel occurred when crack originating from notch tip. This was followed by a gradual increase in steel stress due to stress redistribution, aggregate bridging degradation and fatigue degradation of bond stress between reinforcing bar and concrete.

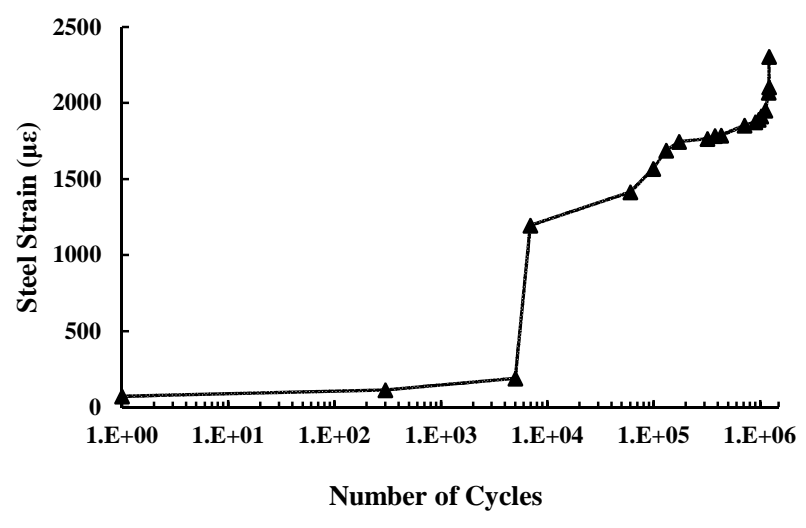

Figure 10: Strain in steel bar at maximum load versus number of cycles of SB-F

In the case of large beam (LB-F), fatigue failure of one bar led to significant stress increase in remaining bars causing failure as explained earlier. Because of overloading, some permanent set in strain in the reinforcing bars was observed. Thus, an overload helps in crack initiating process in bar ribs or defects. At the onset of failure, it was observed that strain in steel started either increasing or decreasing, which resulted in final fatigue failure. 


\subsection{Mid-span deflection and stiffness degradation}

In all beams tested under fatigue loading, initial flexural cracking of concrete caused high stresses in reinforcing bar spanning the crack plane location. It is well known that fatigue causes permanent progressive damage in reinforced concrete beams. As a result, during fatigue loading concrete undergoes cyclic creep in compression zone and tension steel reinforcement experiences cyclic softening. In addition to this, the bond between steel and concrete also deteriorates. Due to the above changes in the materials behavior under fatigue loading, the crack width increases with increase in number of cycles. This increment in crack width leads to a progressive degradation of beam stiffness. The continuous loss of beam stiffness results in increasing mid-span deflections. Figure 11 shows midspan deflection corresponds to maximum fatigue load versus the number of cycles response.

The response of the flexural stiffness corresponding to maximum fatigue load for a typical specimen is shown in Figure 12. A sharp decrease in stiffness is observed due to concrete cracking. After cracking, a gradual loss in stiffness with increase in the number of cycles followed by a sudden drop at the verge of failure.

Application of an overload at $3 \times 10^{5}$ cycles caused a permanent increase in the maximum mid-span deflection as noticed in Figure 13. This overload affected the crack initiation process causing early failure compared to SB-F specimen. In the case of initial overload (SBOF) an overload was applied in the first cycle itself to yield load. This initial overload was followed by the constant amplitude fatigue loading till failure. The midspan deflection versus number of cycles response plotted in Figure 14 shows a similar trend. SB-OF beam attained failure in less number of cycles than beam without any overload.

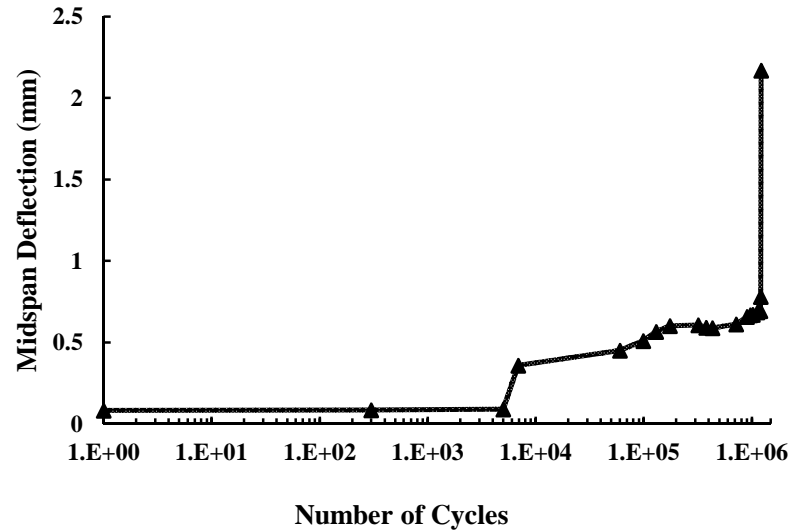

Figure 11: Deflection at maximum load versus number of cycles for SB-F

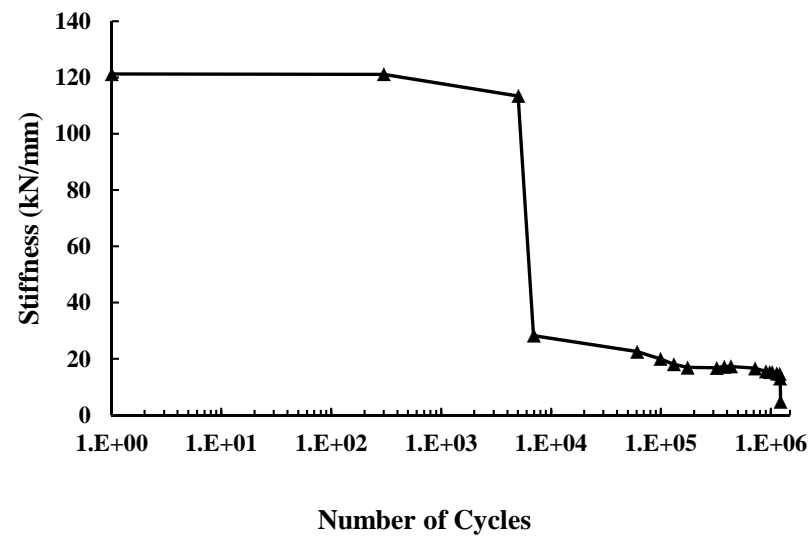

Figure 12: Flexural stiffness versus number of cycles for SB-F

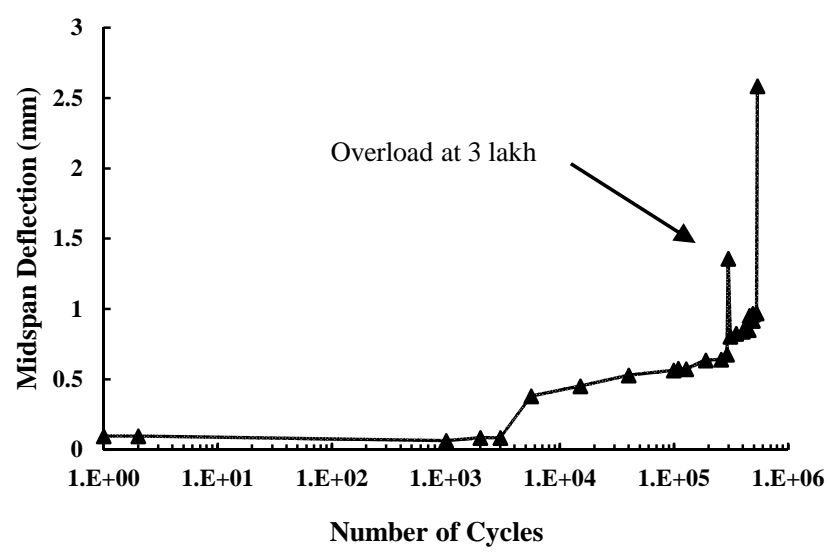

Figure 13: Deflection at maximum load versus number of cycles for SB-FO 


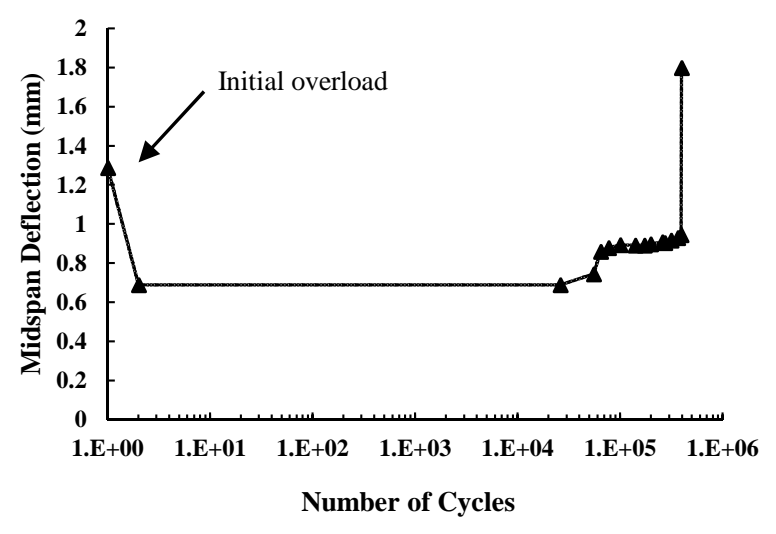

Figure 14: Deflection at maximum load versus number of cycles for SB-OF

\subsection{Concrete softening deterioration}

The transfer of stress across the crack reduces due to the progressive deterioration of material constituents along the crack plane. It was observed during testing, that small quantity of powdered concrete started falling from the crack plane which confirms the softening deterioration. This deterioration of FPZ further increases the stress in steel under constant amplitude fatigue loading. Softening degradation mechanism plays a vital role in the present study because the contribution of concrete in tension has significant influence in the behavior of lightly RC beams.

\subsection{Stress redistribution}

Lightly RC beams tested under monotonic flexure loading failed due to rupture of tension reinforcing steel bars. The response of load versus mid-span deflection in Figure 4 showed ductile under reinforced beam behavior. In fatigue, the extreme compressive top fibers of concrete degrade because of under reinforced beam behavior. With the increase in number of cycles, concrete in compressive zone triggers redistribution of stress along the depth. Because of this redistribution of compressive stresses in concrete and in order to maintain equilibrium, the reinforcing steel bars has to support increments in tensile loads. This stress redistribution causes a further increase in steel strain.

\subsection{Effect of overload}

The applied overload was 54\% more than the maximum constant amplitude of fatigue loading. Initial overload decreased fatigue life by $67 \%$ and an intermediate overload by $48 \%$. Results show that an overload helps in nucleation of crack in steel bar, thereby accelerating crack initiation phase. In Figure 13 it can be observed that intermediate overload caused a residual mid-span deflection which confirms the permanent change in material properties. Similar behavior can be noticed in case of initial overload from Figure 14. After the application of an initial overload no further crack growth was noticed.

\subsection{Effect of size}

It is observed from Table 3 that the number of cycles required for concrete cracking differs slightly with size. For the same applied stress range in the reinforcing steel, the fatigue life of lightly RC beams varies with size. Fatigue failure of large size beam LB-F was significantly lesser than small and medium size beams. In the case of small size beam (SB-F) presence of a defect or inclusion in reinforcing bar nearby the notch dominated the failure. In LB-F beam failure of one in four reinforcing bars resulted in final failure. It is observed that more number of bars can decrease fatigue life.

In lightly RC beams, single predominant crack causes strain localization. In LB-F beam, the reinforcing bars are located away from the neutral axis depth compared to the small and medium beam. Thus, strain localization and steel bar located away from the neutral axis cause local bending in the bar at cracking location in concrete. This local bending in reinforcing bar accelerated crack initiation and propagation process causing relatively shorter fatigue life.

\section{SUMMARY OF FATIGUE TESTS}

Observations from fatigue tests on beams indicated that, during initial cycles prior to concrete cracking, flexural bond ensured transfer of forces from concrete to steel. The flexural crack initiated after few thousand 
cycles as a result of high tensile stress in concrete. Near the cracks, stresses in steel bar increased compared to adjacent sections. High stress concentration near the ribs or inclusion or defects at cracked concrete plane triggers the process of crack initiation in steel bar.

With the increase in fatigue cycles, the strain also found increased. Concrete softening deterioration, flexural bond deterioration and compressive stress redistribution in concrete are responsible for increased strain in steel bar. Strain gauge results were also indicated an increase in the stress in the tensile steel reinforcement under constant amplitude fatigue loading (Figure 10). Under further repetitive cycles at the onset of failure, both reduction and increment in the measured steel strains were observed. In addition, a sudden increase in crack length and crack width were also noticed causing brittle fatigue fracture of reinforcing bar.

Results confirmed that overload accelerated the crack initiation process. Overload also affected the crack length and fatigue life of RC beam. Local bending in reinforcing bars decreases the fatigue life by increasing the rate of crack initiation and propagating process.

\section{CONCLUSIONS}

The following conclusions can be drawn from the experimental observations.

1. Any overload accelerates the crack initiation process on a structural member and reduces its fatigue life.

2. Initial overload reduced fatigue life by $67 \%$ and intermediate overload by $48 \%$.

3. The fatigue life of lightly reinforced concrete beam is size dependent.

4. Deceleration phase in the fatigue crack growth in concrete has a strong influence on fatigue life of lightly RC beams.

\section{REFERENCES}

[1] Helgason, T., and Hanson, J.M. 1974. Investigation of Design Factors Affecting Fatigue Strength of Reinforcing Bars-
Statistical Analysis. ACI Special Publications, Fatigue of Concrete, SP4106: pp. 107-138.

[2] Tilly, G.P. 1979. Fatigue of Steel Reinforcement Bars in Concrete: A Review Fatigue of Engineering Materials and Structures, Vol, 2, pp. 251-268.

[3] Tilly, G.P and Moss, D.S. 1982. Long Endurance Fatigue of Steel Reinforcement.

Proc., IABSE Coll.37: Lausanne, Switzerland, pp. 229-238.

[4] Shah, S. P., Ed., 1982, Fatigue of Concrete Structures, ACI SP-75, American Concrete Institute, Detroit.

[5] Moss, D.S. 1982. Bending Fatigue of High-Yield Reinforcing Bars in Concrete. TRRL Supplementary Rep. 748., Transport and Road Research Laboratory; Crowthome, UK.

[6] Chen, C., and Cheng, L. 2014. Cohesive Model-Based Approach for Fatigue Life Prediction of Reinforced-Concrete Structures Strengthened with NSM FRP. $J$. Compos.Constr.18(2).10.1061/ (ASCE) CC.1943-5614.0000446, 04013042.

[7] Sain, T., and Kishen, J. M. C. 2008. Fracture Stability and Residual Strength Assessment of Reinforced Concrete Beam. Mater. Struct., 41(8), pp. 1451-1463.

[8] Peerapong, S., and Matsumoto, T. 2006. Fatigue Analysis of RC Slabs and Repaired RC Slabs Based on Crack Bridging Degradation Concept. J. Struct. Eng..132: pp. 939-948.

[9] Kolluru, S.V., Neil, E.F.O., Popovics, J.S and Shah, S.P. 2000. Crack Propagation in Flexural Fatigue of Concrete J. Eng. Mech.126: pp. 891-898.

[10]Bazant, Z.P., and Xu, K. 1991. Size Effect in Fatigue Fracture of Concrete. ACI Material Journal, 88 (4), pp. 390-399.

[11]Bazant, Z.P., and Schell, W.F. 1993. Fatigue fracture of High Strength Concrete. ACI Material Journal, 90 (5), pp. 472-478.

[12]Zhang, J., Li, V.C and Strang, H. 2001. Size Effect on Fatigue in Bending of Concrete Journal of Materials in Civil Engineering, ASCE, 13(6), pp. 446-453.

[13] ACI Committee 215. 1997. Considerations 
for Design of Concrete Structures Subjected to Fatigue Loading. Proc., American Concrete Institute, Farmington Hills.

[14] Soltani, A., Harries, K., Shahrooz, B., Russell, H., and Miller, R. 2012. Fatigue Performance of High-Strength Reinforcing Steel. J. Bridge Eng., 061/(ASCE)BE.1943- 5592.0000281, pp. 454-461.

[15] Corley, W. G., Hanson, J. M., and Helgason, T. 1978. Design of Reinforced Concrete for Fatigue. Res. and Devel. Bulletin RD059.01D, PCA.

[16]Willenborg, J., Engle, R.M., Wood, H.A. 1971. A Crack Growth Retardation Model Using an Effective Stress Concept. Air Force Flight Dynamic Laboratory, Dayton, Report, AFFDL-TR71-1.

[17]Wheeler, O.E. 1972. Spectrum Loading and Crack Growth. Journal of Basic Engineering, 94, pp. 181-186.

[18] Slowik, V., Plizzari, G., and Saouma, V. 1996. Fracture of Concrete under Variable Amplitude Loading. ACI Mater J;93(3): pp 272-283.

[19]AASHTO. 2007. LRFD Bridge Design Specifications, 4th Ed., Washington DC.

[20]Canadian Standards Association. 2000. Canadian Highway Bridge Design Code. CAN/CSA- S6- 00, Rexdale, Ont., Canada.

[21]CEB-FIP Model Code. 2010. Vol 1 and Vol.2 Thomas Telford, London.

[22]Papakonstantinou, C. G., Michael, F. P., and Kent, A. H. 2001. Fatigue Behavior of RC Beams Strengthened With GFRP Sheets. J. Compos. Constr..5: pp. 246-253.

[23]Zhao, L. 2005. Characterizations of RC Beams Strengthened with Carbon Fiber Sheets. Ph.D. thesis, University of Alabama, Tuscaloosa, AL, USA; pp. 197.

[24]RILEM. 1985. TC 50-FMC, Fracture Mechanics of Concrete, Determination of the Fracture Energy of Mortar and Concrete by means of Three-Point Bend Tests on Notched Beams, RILEM Recommendation, Materials and Structures, V.18, No. 16, pp. 287-290.

[25]Mallet, G. 1991. Fatigue of Reinforced Concrete. HMSO, London. 